\title{
Modelling the Diffusion of Investment Decisions on Modular Social Networks
}

\author{
Xiaokang Cheng $(\mathbb{D}$ and Narisa Zhao \\ Institute of Systems Engineering, Dalian University of Technology, Dalian 116023, China \\ Correspondence should be addressed to Xiaokang Cheng; chengxk@mail.dlut.edu.cn and Narisa Zhao; nmgnrs@dlut.edu.cn
}

Received 9 July 2019; Accepted 15 February 2020; Published 7 May 2020

Academic Editor: Michele Scarpiniti

Copyright ( 2020 Xiaokang Cheng and Narisa Zhao. This is an open access article distributed under the Creative Commons Attribution License, which permits unrestricted use, distribution, and reproduction in any medium, provided the original work is properly cited.

\begin{abstract}
In the financial market, information and investment behaviors disseminate in investor social networks, and different contagion patterns may cause diverse investment trends. Prior studies have investigated the impact of investor social networks, but few have considered community structure. In this paper, we study the impact of the community structure of investor social networks on the diffusion of internet investment products. A two-stage diffusion model is proposed, and the clustering coefficient and modularity of an investor social network are considered. The results show that both modularity and the clustering coefficient have an impact on the diffusion velocity and scale and that the impact is most evident at the stage of explosive growth. The negative influence of a large modularity can be hardly mitigated by adjusting other factors. Furthermore, a decrease in modularity and an increase in the clustering coefficient can better facilitate diffusion when the temporary investment rate is high and can partly offset the negative impact of information discarding and divestment.
\end{abstract}

\section{Introduction}

The financial market is a complex system in which contagious behavior among investors can result in investment trends in a specific financial product. With the development of online payment technologies and online social platforms, the Internet investment product market has become an important part of the financial market and has drawn attention over time.

An Internet investment product is a personal investment product issued by financial institutions and allows for purchasing on online platforms. A fixed or a nonfixed return is expected according to the underlying contract. Since Alipay launched the online investment product YuE Bao in June 2013, the market for Internet investment products has witnessed prosperity and challenges. Take P2P (Peer-topeer) lending for example, according to data from a website portal for online loans and investments [1], at the end of 2015 , there were 3464 P2P lending platforms, of which over 85\% were launched during 2013-2015. However, due to lack of regulations and supervision, a number of speculative platforms were exposed, and only 2586 were still actively operating in 2016. This number has continued to decrease, and only 935 remained liable by April 2019. In regard to the management of an Internet investment product, understanding the diffusion pattern of investment demand can be useful in risk management.

From the perspective of investors, when faced with an unfamiliar online investment product, people may imitate other investors' behavior [2-4] due to information asymmetry [5, 6] and for psychological reasons [7]. Information and investment behavior may therefore spread epidemically on investor social networks. The community structure of investor social networks can be one of the crucial factors that influences the diffusion dynamics of an Internet investment product. Accordingly, it is vital to understand the diffusion process in modular investor networks.

In this paper, we propose a model to study the diffusion patterns of Internet investment products that consider the community structure of investor social networks. The rest of this paper is organized as follows. In Section 2, we provide a literature review on featured investor peer effects and the 
contagion mechanism in the financial market. In Section 3, we introduce a diffusion model considering the impact of modularity and clustering coefficient of investor social network, and the theoretical derivation is presented. The analytical solutions for all types of nodes in the network are given. In Section 4, we first generate a simulation to examine the accuracy of our model. Then, a numerical analysis is performed to explore the network dynamics under varying parameters. Section 5 summarizes the main findings.

\section{Literature Review}

Community structure demonstrates a profound effect on diffusion pattern in modular networks [8-10]. In the field of behavioral finance, extensive literature has suggested that investors with strong social ties or in geographical proximity are prone to behave similarly, implying that investor network exhibits the features of modular networks. In [11], the authors discovered that fund managers in same city tend to adopt similar trading strategies, regardless of the location of the stock. Such tendency is more significant when they reside in the same neighborhood, especially when they share similar ethnicity [12]. In [13], the authors found that stock market participation is correlated among extended family members, and in [14], the author indicated that the investment decision of a household is affected by its siblings. Although the existence of investor networks' community structure has been implied, the mechanism of how it impacts the diffusion of Internet investment products is still to be unveiled.

To explain the contagion mechanism in the financial market, epidemic models stemming from the SIR model have been applied in prior studies. In [15], a questionnaire survey was conducted to study the spread of investment interest of individual stocks, and the survey evidence appeared to support the epidemic model proposed by the authors. An epidemic model was introduced in [16] to investigate the existence of social influence on stock trading, and the authors found that social trading predicts stock returns. Lu et al. [17] proposed an agent-based financial stock-price model with an epidemic interaction mechanism whose validity was examined by empirical research. The proposed model was proven to be efficient in regenerating crucial features on the real stock market. In [18], the authors proposed a bilayer network model to investigate the interaction mechanism between information spread and credit risk contagion. In [19], based on the epidemic spread of price linkage rumors, the authors explored the contagion of investor risk and discovered global and local effect of investor heterogeneity.

In regard with the diffusion of investment decisions of Internet investment products, in [20], the author observed the epidemic diffusion of a Ponzi scheme caused by word-ofmouth communication, and in $[21,22]$ the authors explored the diffusion features of a Ponzi scheme using epidemic mechanism, where potential investors, investors and divestors were considered. In [23], the authors introduced a two-stage epidemic diffusion model of an Internet investment product, where the spread of information and investment decisions were studied. In this paper, we extend Zhao et al.'s work [23] and apply the epidemic contagion mechanism to examine how a network community structure may influence the dynamics of investment trends.

\section{Theoretical Framework}

The information of an Internet investment product diffuses in investor social networks, and information recipients decide whether to adopt the product. Based on [23], we classify all entities in a network into six categories. The twostage diffusion process of an investment decision is demonstrated in Figure 1. Each vertex in Figure 1 represents one state of an entity. The explanation of each state is presented as follows:

UP (Uninformed potential investor): The node has not received the information about the product and will receive the information upon contact with the node that holds the information, i.e., the P, I, and RI nodes. The contact and information transmission may occur in the same community or in different communities.

$\mathrm{P}$ (Informed potential investor): The node has received the information but has not decided whether to adopt the product.

DS (Discarded): The node discards the information and will never become interested again, i.e., the node will never participate the information spread process again.

I (Temporary investor): The node with the information decides to make a temporary investment.

RI (Regular investor): The node decides to make a regular investment in the product and will not withdraw the investment within the given time.

$\mathrm{D}$ (Divestor): The node withdraws all principal and returns and never participates in the diffusion process again.

UP-P-DS is the diffusion of awareness. P-I-D/RI implies the adoption status of a product. States DS, RI and D are absorbing states; i.e., the nodes will no longer leave these states once they are entered. We use $X_{i}(t), Y_{i}(t)$, and $\mathrm{DS}_{i}(t)$ to indicate the number of P, UP, and DS nodes in community $i$ at time $t$, respectively, and $\mathrm{I}_{\mathrm{i}}(\mathrm{t}), \mathrm{R}_{\mathrm{i}}(\mathrm{t})$, and $\mathrm{D}_{\mathrm{i}}(\mathrm{t})$ to denote the number of $\mathrm{I}, \mathrm{RI}$, and $\mathrm{D}$ nodes in community $i$ at time $t$, respectively.

We define the indicator function $\eta_{j}(t,(t+\Delta t))$ to denote whether a UP node transfers to a $\mathrm{P}$ node during $[t,(t+\Delta t)] . \eta_{j}(t,(t+\Delta t))=1$ implies that the transfer happens; otherwise, $\eta_{j}(t,(t+\Delta t))=0$. Similarly, we use $\omega_{j}(t,(t+\Delta t))$ to denote whether the information is discarded during $[t,(t+\Delta t)] . \omega_{j}(t,(t+\Delta t))=1$ suggests that the $\mathrm{P}$ node discards the information; $\omega_{j}(t,(t+\Delta t))=0$ suggests the opposite. Next, we denote $\varphi_{j}(t,(t+\Delta t))$ as the indicator of whether a $\mathrm{P}$ node decides to make a temporary investment. $\varphi_{j}(t,(t+\Delta t))=1$ implies that a $\mathrm{P}$ node becomes an I node during $[t,(t+\Delta t)]$; otherwise, $\varphi_{j}(t,(t+\Delta t))=0$. We denote $\{\cdot(t)\}$ as the set of all nodes inside state. Thus, we have the following: 


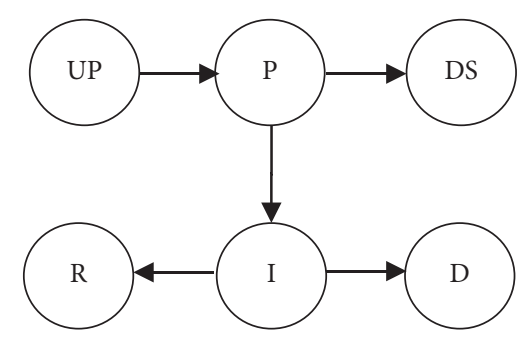

FIgURE 1: The diffusion process of an Internet investment product.

$$
\begin{aligned}
X_{i}(t+\Delta t)= & X_{i}(t)+\sum_{j \in\left\{Y_{i}(t)\right\}} \eta_{j}(t,(t+\Delta t)) \\
& -\sum_{j \in\left\{X_{i}(t)\right\}} \omega_{j}(t,(t+\Delta t)) \\
& -\sum_{j \in\left\{X_{i}(t)\right\}} \phi_{j}(t,(t+\Delta t)) .
\end{aligned}
$$

We use a clustering coefficient $C_{i}$ to represent the connection density inside community $i$ and modularity $Q$ to indicate the connection density between two communities. According to Zhao and Cui et al's work[9], we assume that $C_{i}$ is generated by a normal distribution with mean value $b$ and standard deviation $\sigma$, i.e., $C_{i} \sim\left(b, \sigma^{2}\right)$. We denote information spreading probability as $p_{s}$, which is positively correlated to $C_{i}$ when the information sender and recipient are in the same community, and inversely correlated to $Q$ when they reside in different communities. We assume that either in the same community or between different communities, $p_{s}$ is drawn from an exponential distribution. Thus, we have the following:

$$
p_{s}=\left\{\begin{array}{l}
1-e^{-\alpha(1-Q) t}, i \neq j, \\
1-e^{-\alpha C_{i} t}, \quad i=j, i, j \in[1, \ldots, K],
\end{array}\right.
$$

where $i / j$ denote the $i$ th $/ j$ th community, $K$ indicates the total number of communities, and $\alpha$ is the adjusting parameter. Therefore, the probability that a UP node adopts the information and becomes a $\mathrm{P}$ node can be described as follows:

$$
\begin{aligned}
\mathrm{P}\left(\eta_{j}(t+\Delta t)=1\right) & =1-\left(e^{-\alpha C_{i} \Delta t}\right)^{X_{i}(t)+Y_{i}(t)+R_{i}(t)} \cdot \prod_{j=1, \ldots, K, j \neq i}\left(1-\left(e^{-\alpha(1-Q) \Delta t}\right)\right)^{X_{j}(t)+Y_{j}(t)+R_{j}(t)} \\
& =1-\exp \left(-\alpha\left(C_{i}\left(X_{i}(t)+Y_{i}(t)+R_{i}(t)\right)\right)\right) \Delta t+(1-Q) \sum_{j=1, \ldots, K, j \neq i}\left(\left(X_{i}(t)+Y_{i}(t)+R_{i}(t)\right) \Delta t\right) .
\end{aligned}
$$

To simplify the equation, let $X_{i}(t)+Y_{i}(t)+$ $R_{i}(t)=Z_{i}(t)$; we then have the following:

$$
\begin{aligned}
\mathrm{P}\left(\eta_{j}(t+\Delta t)=1\right)=1 & -\exp \left(-\alpha\left(C_{i} Z_{i}(t) \Delta t+(1-Q)\right)\right) \\
& \cdot \sum_{j=1, \ldots, K, j \neq i}\left(Z_{j}((t) \Delta t)\right) .
\end{aligned}
$$

Next, we focus on the transition process from state $\mathrm{P}$ to states DS and I. We denote $\beta$ as the information rejection rate and $\gamma$ as the investment rate, and we assume that transmission probability follows exponential distribution. Thus, the transition probabilities from $\mathrm{P}$ to $\mathrm{I}$ and from $\mathrm{S}$ to DS are shown in equations (5) and (6) as follows:

$$
\begin{aligned}
& \mathrm{P}\left(\omega_{j}(t+\Delta t)=1\right)=1-e^{-\beta \Delta t}, \\
& \mathrm{P}\left(\varphi_{j}(t+\Delta t)=1\right)=1-e^{-\gamma \Delta t} .
\end{aligned}
$$

Therefore,

$$
\begin{aligned}
E\left(X_{i}(t+\Delta t)\right)= & E\left(X_{i}(t)\right)+E\left(Y_{i}(t)\right) E\left(\eta_{j}(t+\Delta t)\right) \\
& -E\left(X_{i}(t)\right) E\left(\omega_{j}(t+\Delta t)\right) \\
& -E\left(X_{i}(t)\right) E\left(\phi_{j}(t+\Delta t)\right) .
\end{aligned}
$$

Taking limitations on both sides [21], we have the following:

$$
\begin{aligned}
\lim _{\Delta t \longrightarrow 0} & \frac{E\left(X_{i}(t+\Delta t)\right)-E\left(X_{i}(t)\right)}{\Delta t} \\
= & \lim _{\Delta t \longrightarrow 0} \frac{E\left(Y_{i}(t)\right) E\left(1-\exp \left(-\alpha\left(C_{i} Z_{i}(t) \Delta t+(1-Q) \sum_{j=1, \ldots, K, j \neq i} Z_{j}(t) \Delta t\right)\right)\right)}{\Delta t} \\
& -\lim _{\Delta t \longrightarrow 0} \frac{E\left(X_{i}(t)\right) E\left(1-e^{-\beta \Delta t}\right)}{\Delta t}-\lim _{\Delta t \longrightarrow 0} \frac{E\left(X_{i}(t)\right) E\left(1-e^{-\gamma \Delta t}\right)}{\Delta t} \\
\Delta E\left(\dot{X_{i}}(t)\right)= & \alpha E\left(Y_{i}(t)\right)\left(C_{i} Z_{i}(t) \Delta t+(1-Q) \sum_{j=1, \ldots, K, j \neq i} Z_{j}(t)\right)-(\beta+\gamma) E\left(X_{i}(t)\right) .
\end{aligned}
$$


Similarly,

$E\left(\dot{Y_{i}}(t)\right)=-\alpha E\left(Y_{i}(t)\right)\left(C_{i} Z_{i}(t) \Delta t+(1-Q) \sum_{j=1, \ldots, K, j \neq i} Z_{j}(t)\right)$

$$
E\left(\dot{D S}_{i}(t)\right)=\beta E\left(X_{i}(t)\right)
$$

After a temporary investment is made, an investor will decide whether to make a regular investment or to quit. We use the indicator function $\sigma_{j}(t,(t+\Delta t))$ to define whether the divestment occurs during $[t,(t+\Delta t)] . \sigma_{j}(t,(t+\Delta t))=$ 1 suggests that the temporary investor becomes a divestor; otherwise, $\sigma_{j}(t,(t+\Delta t))=0$. Similarly, we use $\theta_{j}(t,(t+\Delta t))$ to indicate whether an I node becomes an RI node during $[t,(t+\Delta t)] \cdot \theta_{j}(t,(t+\Delta t))=1$ implies that the I node changes to the state of the RI node; otherwise, $\theta_{j}(t,(t+\Delta t))=0$.
Thus, the dynamics of the number of I nodes can be described as follows:

$$
\begin{aligned}
I_{i}(t+\Delta t)= & I_{i}(t)+\sum_{j \in\left\{X_{i}(t)\right\}} \phi_{j}(t,(t+\Delta t)) \\
& -\sum_{j \in\left\{I_{i}(t)\right\}} \sigma_{j}(t,(t+\Delta t))-\sum_{j \in\left\{X_{i}(t)\right\}} \theta_{j}(t,(t+\Delta t)) .
\end{aligned}
$$

Assuming that the transition probabilities from state I to $\mathrm{RI}$ and $\mathrm{W}$ are drawn from an exponential distribution with parameters $\lambda$ and $\mu$, respectively, we have the following:

$$
\begin{aligned}
& \mathrm{P}\left(\theta_{j}(t,(t+\Delta t))=1\right)=1-e^{-\lambda \Delta t} \\
& \mathrm{P}\left(\sigma_{j}(t,(t+\Delta t))=1\right)=1-e^{-\mu \Delta t} .
\end{aligned}
$$

Taking the limit of the expectation of equation (11), we have the following:

$$
\begin{aligned}
\lim _{\Delta t \rightarrow 0} \frac{E\left(I_{i}(t+\Delta t)\right)-E\left(I_{i}(t)\right)}{\Delta t}= & \lim _{\Delta t \rightarrow 0} \frac{E\left(X_{i}(t)\right) E\left(1-e^{-\gamma \Delta t}\right)}{\Delta t}-\lim _{\Delta t \rightarrow 0} \frac{E\left(I_{i}(t)\right) E\left(1-e^{-\lambda \Delta t}\right)}{\Delta t} \\
& -\lim _{\Delta t \rightarrow 0} \frac{E\left(I_{i}(t)\right) E\left(1-e^{-\mu \Delta t}\right)}{\Delta t} \Rightarrow E\left(I_{i}(t)\right)=\gamma E\left(X_{i}(t)\right)-(\mu+\lambda) E\left(I_{i}(t)\right) .
\end{aligned}
$$

Similarly,

$$
\begin{aligned}
& E\left(\dot{R_{i}}(t)\right)=\lambda\left(I_{i}(t)\right), \\
& E\left(\dot{D_{i}}(t)\right)=\mu E\left(I_{i}(t)\right) .
\end{aligned}
$$

\section{Simulation and Numerical Results}

In this section, intuitive images of the transition processes of all types of nodes are presented in Figure 2 and a simulation is conducted to examine the accuracy of the model. We assume that there are 10000 entities evenly distributed across 5 communities. At the initial time, only one node in the first community holds the information. Then, the information is spread when this node contacts other nodes in the same community or in different communities. The transmission dynamics of all types of nodes are illustrated in Figure 2. The parameter settings in Figure 2 are default settings in the following sections.

From Figure 2(a), we can see that the number of uninformed nodes decreases sharply at the very beginning. Accordingly, the number of $\mathrm{P}$ nodes increases rapidly to a peak value and then reduces to 0 at a relatively gentle speed. Such reduction is caused by information rejection and investment decisions. The number of temporary investor nodes increases to a peak and then decreases due to the transition to regular investment and divestment. The percentage of RI nodes remains at approximately $27 \%$ during the diffusion, which is in agreement with the percentage of valid accounts of open-ended fund investors at the end of 2015 [24]. Figure 2(b) shows a more clear curve of the dynamics of regular investors, where $r(T)$ denotes the percentage of $R(T)$. The $\mathrm{S}$-shaped diffusion curve is consistent with the diffusion curve of financial innovation discovered in [25].

Next, we run a simulation according to the diffusion mechanism in our model to test its accuracy. We use the number of regular investors in the comparison, and the result is demonstrated in Figure 3. To eliminate occasional errors, we calculate the value of each period 5 times and take the mean value as the simulation result. At the very beginning of the dissemination, the total number of RI nodes is very small, thus a small difference in the number of RI nodes can cause a large error. Therefore, we exclude the first two nodes in the error calculation; the error of the remaining nodes is $2.91 \%$, suggesting that our model is accurate in describing the diffusion process.

4.1. Effect of Modularity. The value of modularity $Q$ implies the connection density between two different communities. A large $Q$ suggests a high level of partition and therefore a small transition probability between two communities. The impact of modularity is illustrated in Figure 4.

As shown in Figure 4(a), the value of $r(t)$ is inversely related to $Q$. Such alleviation becomes increasingly significant with the increase in $Q$. Within the given time scale, only the increasing speed is influenced by $Q$ when $Q$ is smaller than 0.7. As $Q$ continues to increase, the final proportion of regular investor nodes will be affected as well.

In Figure 4(b), we can see that the increase in $Q$ leads to an evident decrease in RI when the total duration is short. 


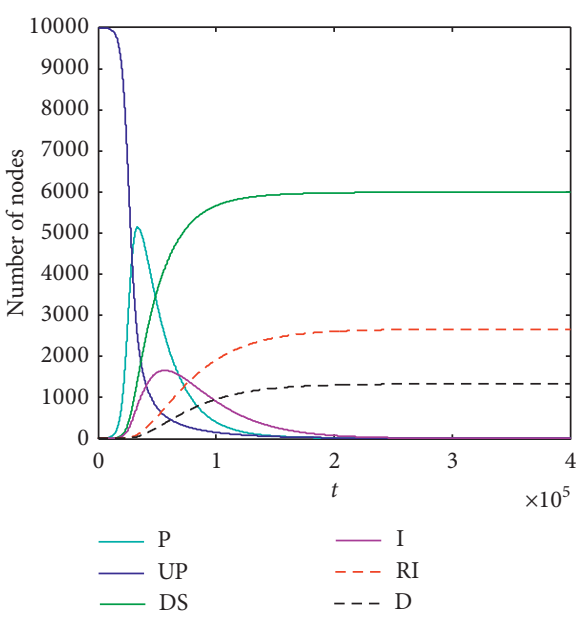

(a)

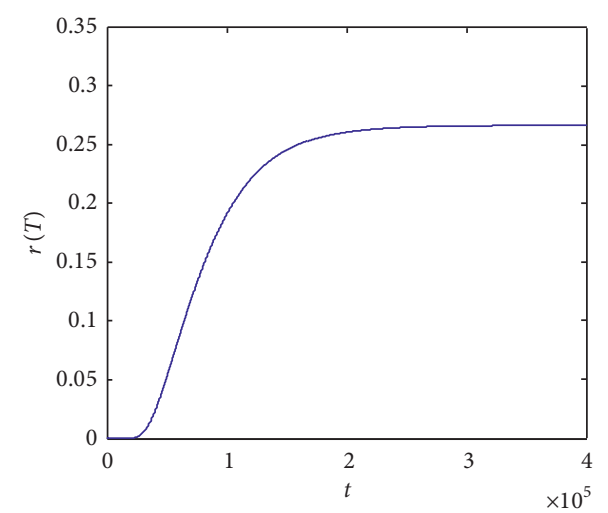

(b)

Figure 2: (a) The number of nodes in the six states versus time with $T=4 \times 10^{5} \mathrm{~s}$. (b) The proportion of regular investor nodes against time. Other parameters are set as follows: $\alpha=1 \times 10^{-7}, \beta=3 \times 10^{-5}, \gamma=2 \times 10^{-5}, \mu=1 \times 10^{-5}, \lambda=2 \times 10^{-5}, T=4 \times 10^{5}, Q=0.3, C_{i} \sim N\left(0.9,0.1^{2}\right), K=5$.

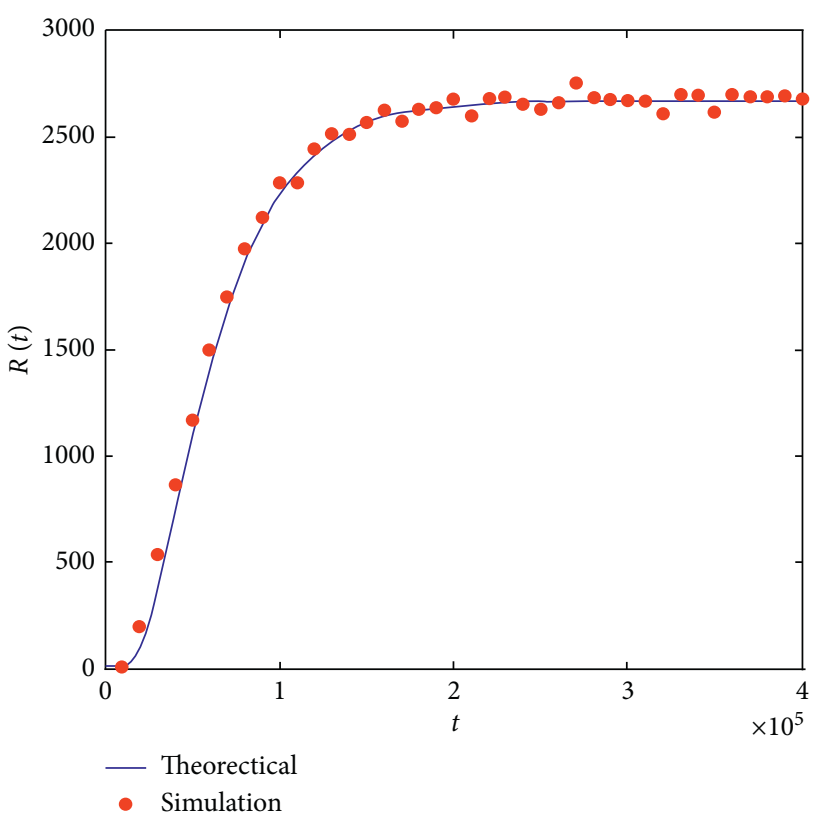

FIGURE 3: Comparison between theoretical results and simulation.

When the propagation time goes beyond 20000, the reduction effect is mitigated when $Q$ is smaller than 0.9. When $Q$ increases to a point close to 1 , there is a sharp drop in $r(T)$. Actually, a very large $Q$ suggests a very low probability that the information will be transmitted between communities; thus, the diffusion is halted before it reaches other communities.

In summary, modularity $Q$ mainly changes the spread velocity when $T$ is long enough. When $Q$ is close to 1 , it leads to a dramatic decline in $r(T)$. Such results indicate that to achieve a rapid propagation, a decrease in modularity in the network would be helpful. Accordingly, in the case of a fraudulent investment, an extreme increase in modularity would be useful for blocking the information regarding the scheme.
4.2. Effect of the Clustering Coefficient. The clustering coefficient $C_{i}(i=1, \ldots, K)$ indicates the connection density inside community $i$. As stated in Section 2 , we assume that $C_{i}$ follows a normal distribution with a mean value $b$. Figure 5 presents the role of $C_{i}$ in the diffusion pattern of an Internet investment product.

In Figure 5(a), we can see that $C_{i}$ changes both the speed and the outbreak time of the diffusion. The growth of $b$ leads to an acceleration in the diffusion, and such a positive influence is more distinct at the explosive outbreak stage, indicating that strategies to enhance the clustering coefficient should be performed at the explosive growth stage or in advance.

Figure 5(b) shows the final percentage of RI nodes against $b$ with different lifetimes. When $T=10000$, the change in $b$ brings an S-shaped increase in $r(T)$. With the expansion of total lifetime, the curve shows a different trend. $r(t)$ increases rapidly at the beginning and then smoothly afterwards. Such findings suggest that when the propagation lifetime is long enough and the clustering coefficients of the communities are small, an increase in $C_{i}$ would be effective to boost the diffusion of an Internet investment product.

In summary, the increase of clustering coefficient $C_{i}$ results in an earlier and faster outbreak. With the expansion of propagation lifetime, improving $C_{i}$ is more effective to promote the diffusion when $C_{i}$ is small.

4.3. Mutual Effect Analysis. In the last two sections, we studied the individual roles of modularity and the clustering coefficient when other parameters in the diffusion process were constant. Now, we investigate the situation in which the other parameters are dynamic. Figure 6 illustrates the mutual effect of modularity and the temporary investment rate, and the information rejection rate and divestment rate.

In Figure 6, we can see that, under all the given circumstances, the change in $Q$ has little effect on the scale of RI when $Q$ is smaller than 0.7 . When $Q$ varies between 


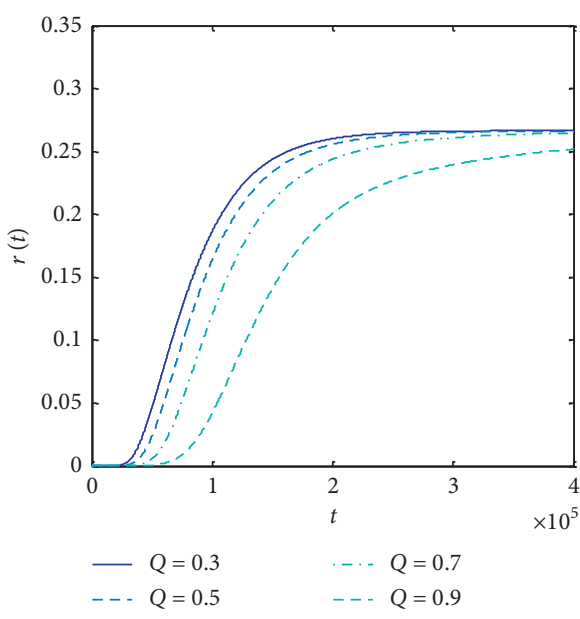

(a)

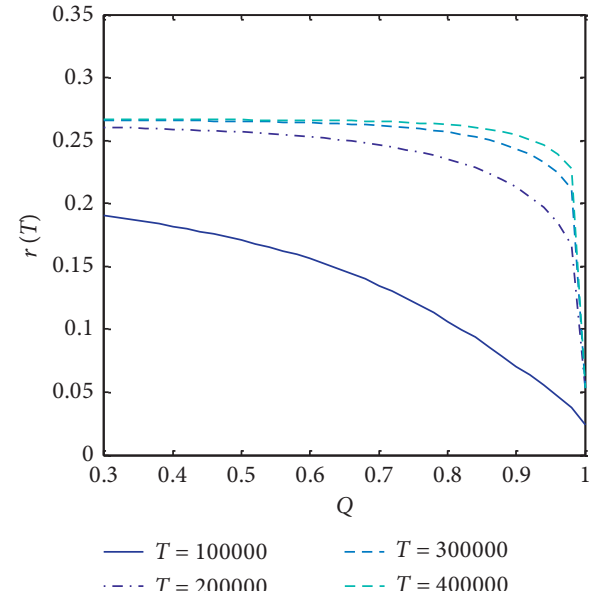

(b)

Figure 4: (a) Proportion of RI nodes against time $\mathrm{t}$ with $b=0.7$ and $Q=0.3,0.5,0.7,0.9$. (b) The final percentage of RI nodes with different lifetimes when $Q$ varies from 0.3 to 1 and $b=0.7$.

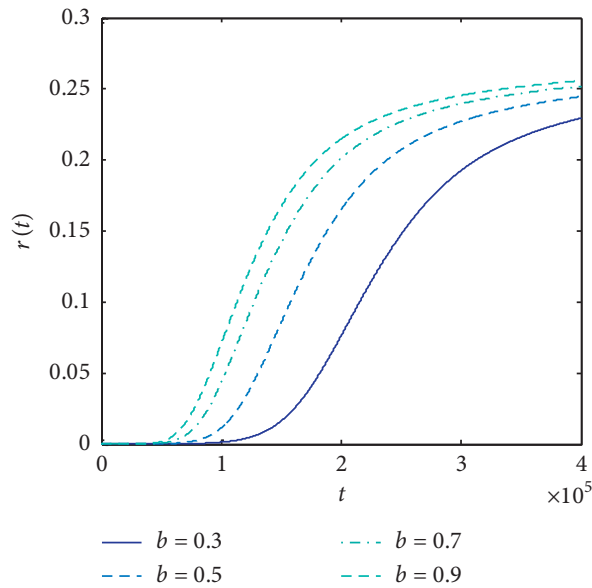

(a)

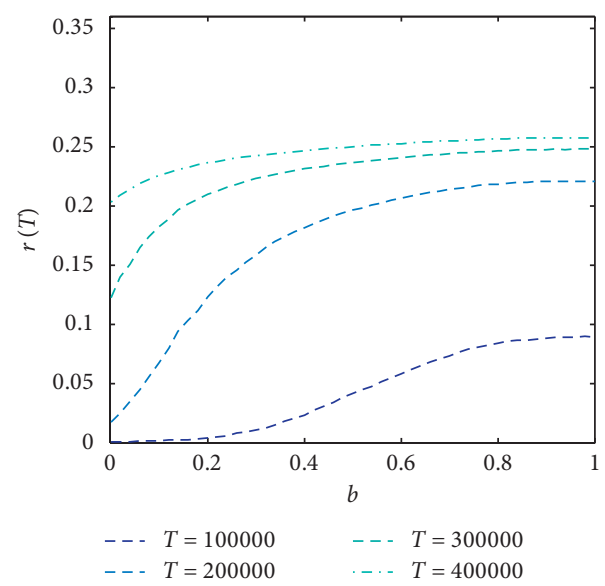

(b)

Figure 5: (a) Proportion of RI nodes with $Q=0.9$ and $b=0.3,0.5,0.7,0.9$. Other settings are the same as the default settings. (b) The final proportion of RI nodes with $\mathrm{b}$ changing from 0 to 1 and $T=100000,200000,300000,400000$, respectively. $Q=0.9$. Other settings are the same as the default settings.

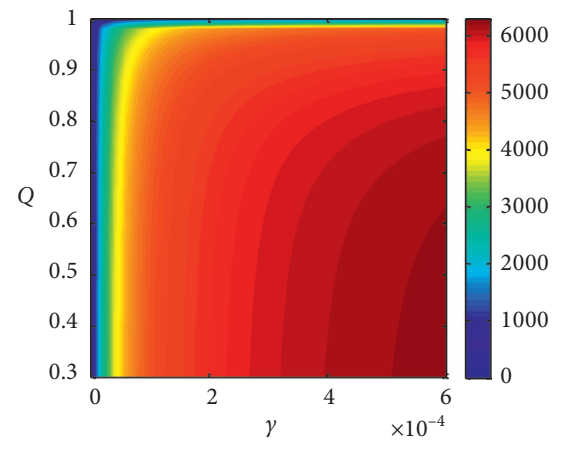

(a)

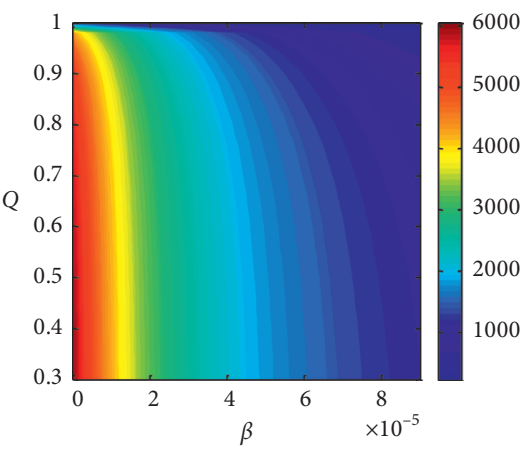

(b)

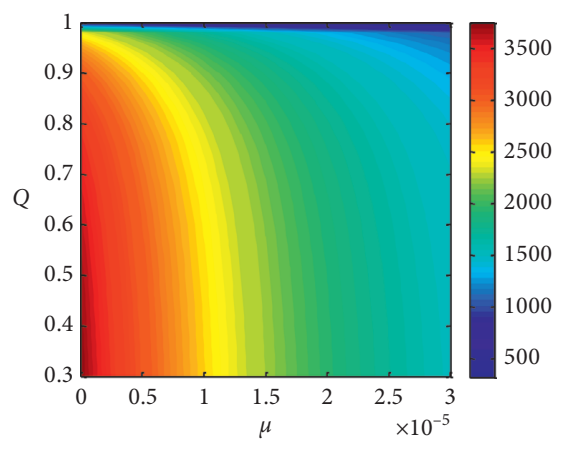

(c)

FIgURE 6: Number of RI nodes with changing $Q$ versus $\gamma, \beta$, and $\mu$, respectively. $b=0.8, T=20000$, and the other settings are the same as the default settings. 


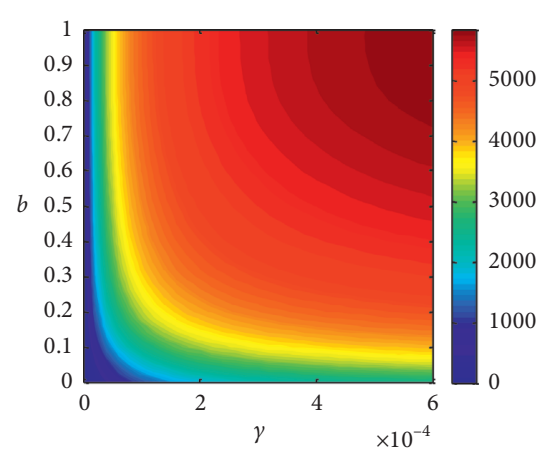

(a)

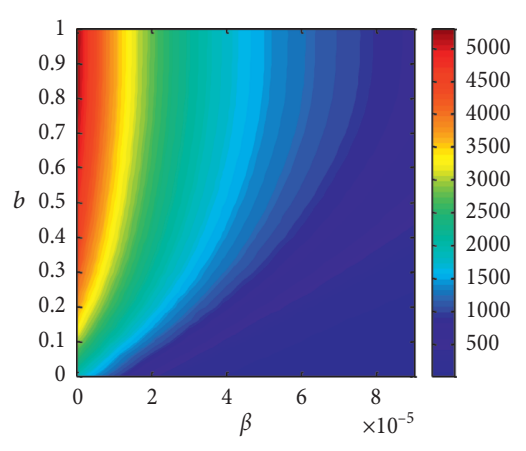

(b)

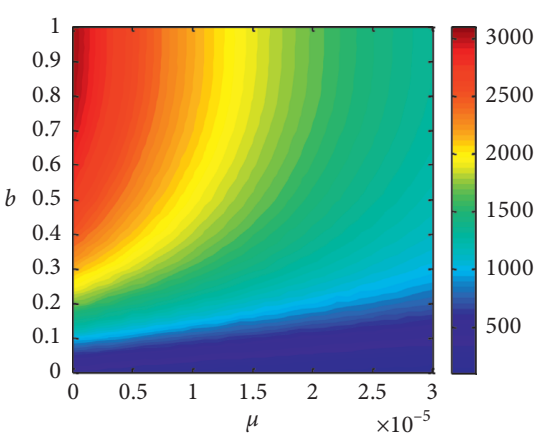

(c)

Figure 7: Number of RI nodes with changing $b$ versus $\gamma, \beta$, and $\mu$, respectively. $Q=0.9, T=20000$, and other settings are the same as the default settings.

0.7 and 1, Figure 6(a) shows that $Q$ becomes increasingly influential with an increase in the temporary investment rate $\gamma$, suggesting that when the temporary investment rate is higher, the same decrement in $Q$ can bring a larger number of RIs. Figure $6(\mathrm{~b})$ implies that the change in $Q$ is decreasingly effective as $\beta$ increases. The same trend can be found in Figure 6(c) as well, but in an alleviated manner, indicating that compared with the negative force of information abandonment, the negative impact of divestment is easier to mitigate by decreasing $Q$. In addition, when $Q$ comes close to 1 , no matter how the other three parameters change, the number of RI nodes is restrained at a low level.

Next, we study the mutual effect of the clustering coefficient and the above three parameters. The results are plotted in Figure 7.

Figure 7(a) shows that the positive effect of $b$ is increasingly significant with the growth in $\gamma$. Figure $7(\mathrm{~b})$ suggests that when the information discarding rate is not too high, its negative effect can be counteracted by an increase in $b$. In Figure 7(c), we can see that the increment in $b$ also covers the deficit caused by divestment, and such mitigation is decreasingly effective as $\mu$ increases. Moreover, when $b$ is smaller than 0.1 , the diffusion scope can be adjusted by improving $\gamma$ or decreasing $\beta$; however, changing $\mu$ can hardly be of help, which suggests that when the connections are sparse inside communities, instead of focusing on cutting the divestment rate, efforts should be made to improve the temporary investment rate or to avoid information discarding.

In summary, the increase of temporary investment rate or the decrease of information rejection rate amplifies the impact of changing network structure. Adjusting the network structure can offset the negative influence of information rejection or divestment, and such phenomenon is more evident with the latter.

\section{Conclusions}

In this paper, we introduce a model to study the impact of an investor community structure on the diffusion of an Internet investment product. Modularity and the clustering coefficient are considered in a two-stage diffusion process. A simulation is conducted, and the accuracy of our model is tested.

Our study suggests that both modularity and the clustering coefficient change the diffusion velocity and scale of an Internet investment product. When modularity comes close to 1, i.e., when the information transmission probability between communities is close to 0 , the diffusion scope severely declines. Second, the positive impact of the clustering coefficient is most evident at the explosive growth stage of diffusion, indicating that measures to improve the clustering coefficient should be taken before this stage. When the promotion lifetime is long enough, the increase in the clustering coefficient is more effective when the coefficient is small. In addition, a decline in modularity or a rise in the clustering coefficient becomes increasingly effective with a rise in the temporary investment rate. Last but not least, the negative force of the information discarding rate and divestment rate can be alleviated by a change in the community structure, and the mitigation of the latter is more significant.

Our study extends Zhao et al.'s study [23] by taking the community structure of investor social networks into consideration. Theoretically, we propose an improved twostage diffusion model to study the diffusion of an Internet investment product in a structured network. Practically, our analysis offers insights for when and how to make the most use of the community structure of investor networks.

In future research, threshold behaviors of the structured investor social network could be considered [20]. In addition, multiple types of investment communities [26], overlapping community structures, or investor social networks with dynamic topology [27] could be studied.

\section{Data Availability}

The main methods in this research are numerical analysis and simulation. The images of numercial analysis are obtained by plot function of Matlab2014a, and the simulation data are random data generanated by Java according to the relevant contagion mechanism. Therefore, the data used to support the findings of this study have not been made available. 


\section{Conflicts of Interest}

The authors declare that they have no conflicts of interest.

\section{Acknowledgments}

This research was supported by the General Program of Natural Science Foundation of China (Grant no. 61471083), Humanities and Social Sciences Research Program of the Ministry of Education of China (Grant no. 14YJA630044), and Scientific and Technological Innovation Foundation of Dalian (Grant no. 2018J11CY009).

\section{References}

[1] https://shuju.wdzj.com/industry-list.html.

[2] R. Z. Heimer, "Peer pressure: social interaction and the disposition effect," Review of Financial Studies, vol. 29, no. 11, pp. 3177-3209, 2016.

[3] E. Y. Chan and N. U. Saqib, "Online social networking increases financial risk-taking," Computers in Human Behavior, vol. 51, no. PA, pp. 224-231, 2015.

[4] K. R. Ahern, R. Duchin, and T. Shumway, "Peer effects in risk aversion and trust," Review of Financial Studies, vol. 27, no. 11, pp. 3213-3240, 2014.

[5] A. V. Banerjee, "A simple model of herd behavior," The Quarterly Journal of Economics, vol. 107, no. 3, pp. 797-817, 1992.

[6] S. Bikhchandani, D. Hirshleifer, and I. Welch, "A theory of fads, fashion, custom, and cultural change as informational cascades," Journal of Political Economy, vol. 100, no. 5, pp. 992-1026, 1992.

[7] S. G. Barsade, "The ripple effect: emotional contagion and its influence on group behavior," Administrative Science Quarterly, vol. 47, no. 4, pp. 644-675, 2002.

[8] J. Wang, N. Yang, Y. Zhang, and Y. Song, "Modeling and simulation of the cascading failure of R\&D network considering the community structure," Physica A: Statistical Mechanics and Its Applications, vol. 522, pp. 43-53, 2019.

[9] N. Zhao and X. Cui, "Impact of individual interest shift on information dissemination in modular networks," Physica A: Statistical Mechanics and Its Applications, vol. 466, pp. 232242, 2017.

[10] R. Zhang and D. Li, "Rumor propagation on networks with community structure," Physica A: Statistical Mechanics and Its Applications, vol. 483, pp. 375-385, 2017.

[11] H. Hong, J. D. Kubik, J. C. Stein, and C. S. Jeremy, "Social interaction and stock-market participation," The Journal of Finance, vol. 59, no. 1, pp. 137-163, 2004.

[12] V. K. Pool, N. Stoffman, and S. E. Yonker, "The people in your neighborhood: social interactions and mutual fund portfolios," The Journal of Finance, vol. 70, no. 6, pp. 2679-2732, 2015.

[13] G. Li, Information Sharing and Stock Market Participation: Evidence from Extended Families, pp. 151-160, Board of Governors of the Federal Reserve System, Washington, D.C., USA, 2009.

[14] K. Tokuoka, "Is stock investment contagious among siblings?" Empirical Economics, vol. 52, no. 4, pp. 1505-1528, 2017.

[15] R. J. Shiller and J. Pound, "Survey evidence on diffusion of interest and information among investors," Journal of Economic Behavior \& Organization, vol. 12, no. 1, pp. 0-66, 1989.
[16] S. Shive, “An epidemic model of investor behavior," Journal of Financial and Quantitative Analysis, vol. 45, no. 1, pp. 169198, 2010.

[17] Y. Lu, J. Wang, and H. Niu, “Agent-based financial dynamics model from stochastic interacting epidemic system and complexity analysis," Physics Letters A, vol. 379, no. 14-15, pp. 1023-1031, 2015.

[18] Q. Qian, Y. Yang, J. Gu, and H. Feng, "Information authenticity, spreading willingness and credit risk contagion - a dual-layer network perspective," Physica A: Statistical Mechanics and Its Applications, vol. 536, p. 122519, 2019.

[19] Y. Dong, J. Wang, and T. Chen, "Price linkage rumors in the stock market and investor risk contagion on bilayer-coupled networks," Complexity, vol. 2019, Article ID 4727868, 21 pages, 2019.

[20] V. Rantala, "How do investment ideas spread through social interaction? Evidence from a ponzi scheme," Journal of Finance, vol. 74, no. 5, pp. 2349-2389, 2019.

[21] A. Zhu, P. Fu, Q. Zhang, and Z. Chen, "Ponzi scheme diffusion in complex networks," Physica A: Statistical Mechanics and Its Applications, vol. 479, pp. 128-136, 2017.

[22] P. Fu, A. Zhu, H. Ni, X. Zhao, and X. Li, “Threshold behaviors of social dynamics and financial outcomes of Ponzi scheme diffusion in complex networks," Physica A: Statistical Mechanics and Its Applications, vol. 490, pp. 632-642, 2018.

[23] N. Zhao, X. Cheng, and X. Guo, "Impact of information spread and investment behavior on the diffusion of internet investment products," Physica A: Statistical Mechanics and Its Applications, vol. 512, pp. 427-436, 2018.

[24] Asset Management Association of China, 2015 China Securities Investment Fund Fact Book, China Financial and Economic Publishing House, Beijing, China, 2015.

[25] A. Marszk and E. Lechman, "Tracing financial innovation diffusion and substitution trajectories. Recent evidence on exchange-traded funds in Japan and South Korea," Technological Forecasting and Social Change, vol. 133, pp. 51-71, 2018.

[26] A. Spelta, A. Flori, and F. Pammolli, "Investment communities: behavioral attitudes and economic dynamics," Social Networks, vol. 55, pp. 170-188, 2018.

[27] M. Oldham, "Understanding how short-termism and a dynamic investor network affects investor returns: an agentbased perspective," Complexity, vol. 2019, Article ID 1715624, 21 pages, 2019. 